\title{
Lifetimes of Energy Levels in Neutral Iron
}

\author{
C. H. Corliss and J. L. Tech \\ Institute for Basic Standards, National Bureau of Standards, Washington, D.C. 20234
}

(August 15, 1967)

\begin{abstract}
Mean radiative lifetimes for 408 energy levels of neutral iron are calculated from the known transition probabilities of 3288 lines of Fe I.
\end{abstract}

Key Words: Atomic spectra, energy levels, iron, iron-lifetimes in, lifetimes in Fe I, radiative lifetimes, spectroscopy.

\section{Introduction}

Let us first briefly review the concept of mean radiative lifetime. Even in the absence of de-excitation caused by such external factors as collisional processes or an ambient radiation field, an atom in an excited state $E_{i}$ will eventually suffer a spontaneous transition to a state of lower energy $E_{j}$ and will emit a photon of energy in accordance with Bohr's energy relation, $h \nu=E_{i}-E_{j}$. The probability per unit time, $A_{i j}$, associated with this type of spontaneous decay is a physical constant for the particular transition and was first described by Einstein [1917]. For a system of $N_{i}$ atoms in an excited state of energy $E_{i}$, we can write the decay rate as

$$
\frac{d N_{i}}{d t}=-\Sigma_{j} A_{i j} N_{i}
$$

the summation being extended over all states $E_{j}$ of energy lower than $E_{i}$. By setting $1 / \tau_{i}=\Sigma_{j} A_{i j}$ and integrating, we obtain

$$
N_{i}=N_{i}^{0} e^{-t / \tau_{i}}
$$

where $N_{i}^{0}$ is the number of atoms in the excited state at time $t=0$. After a time interval equal to $\tau_{i}$, therefore, the number of atoms remaining in the excited level will have been reduced by a factor $1 / e$. This time interval is defined as the mean radiative lifetime of the given energy state and can be measured by a variety of wellestablished experimental methods, as described in the well-known work by Mitchell and Zemansky [1934].
As a result of the connection between transition probabilities ( $A$-values) and oscillator strengths ( $f$-values), namely

$$
A_{i j}=\frac{6.670}{\lambda^{2}} \frac{g_{j}}{g_{i}} f_{j i}
$$

where $g$ is the statistical weight, $2 J+1$, of the level, it is possible to calculate the lifetime of an energy level

$$
\tau_{i}=1 / \Sigma_{j} A_{i j}
$$

provided only that the $f$-values for all possible downward transitions are known. The practicability of this indirect method is seriously limited, however, because for levels other than those yielding simple resonance lines the method requires not only a rather complete knowledge of the energy-level structure of the given atom, but also $f$-values in quantities not usually available. In the case of iron, however, these requirements no longer are insurmountable.

\section{Discussion of the Data}

For a variety of reasons, the spectrum of iron has traditionally been the object of considerable attention. The term analysis is sufficiently advanced that it was possible for Corliss and Bozman [1962] to derive $f$-values for a large number of iron lines by a simple reduction of accurate arc emission intensities. Corliss and Warner [1964, 1966] later extended this work by adding new and fainter lines. For many of the lines, the evaluation of 19 other sets of measurements in 
the literature made it possible for them to arrive at a "best" value for $g f$.

In these previous compilations the calculation of $g f$-values included the application of an empirically determined normalization to put the relative $g f$-values on an absolute scale, the normalization being given as a function of upper energy level for the transition. This function, which was essentially a correction to level populations for departures from a Boltzmann distribution in the arc, reduces the value of $g f$ calculated from line intensities measured in the free-burning arc below that which would have been calculated with a simple Boltzmann factor. The correction affected all lines that arise from upper energy levels lying above $46,000 \mathrm{~cm}^{-1}$, the magnitude of the correction becoming progressively larger as the value of the upper level increased.

Recent investigations by Huber and Tobey [1967], who measured oscillator strengths for Fe I by use of a shock tube, do not support these excitation corrections in the case of Fe I. A similar conclusion may be drawn from the work of Cowley and Warner [1967], who have decided on the basis of a theoretical model for the arc that a single normalization function is not applicable to all spectra but that the function depends on both the ionization potential and the ionization stage of the atom under study. For iron, their work indicates that no correction at all should be applied. In view of these concordant results, we have recalculated $g f$-values for all lines to which the excitation correction had previously been applied. The recalculated $g f$-values, together with other supporting material for the present calculation of lifetimes, are being published separately in a monograph by Corliss and Tech [1967]. That monograph covers the spectral range from 2084 to 9900 angstroms. As demonstrated there, the best available evidence indicates that the data presented in this new compilation do not now suffer from any serious systematic error.

Using these revised data we have calculated a number of intensity-related quantities, including $A$-values, for over 3000 spectral lines, and from these have been able to derive mean radiative lifetimes for over 400 energy levels of neutral iron. In a work of this kind, it is essential that the spectral range considered be sufficiently extensive to contain most of the lines in which the energy of the system is radiated and that no syste- matic error affects the data over that spectral range. We feel that the homogeneity and the extensive wavelength coverage of the present material on iron meets these requirements.

It has thus become possible for the first time to present for a complex atom a fairly comprehensive picture of individual lifetimes for a large number of its energy states. Apart from their own intrinsic interest, these lifetimes can additionally serve as the basis for a computation of the radiation damping constant for any line given in the aforementioned monograph. These constants, required in any calculation of line absorption coefficients for pure radiation damping, are given simply by the sum of the reciprocal lifetimes of the upper and lower energy levels involved in the transition. Thus, for a line produced by the transition $E_{i} \rightarrow E_{j}$, the damping constant will be given by

$$
\Gamma_{i j}=\frac{1}{\tau_{i}}+\frac{1}{\tau_{j}}=\Gamma_{i}+\Gamma_{j}
$$

Of course, in most actual sources, for example those at high temperatures or high particle densities, other factors affecting the lifetimes come into play in fixing the damping constants and must be included in any such calculation of absorption coefficients or line profiles. However, the above expression for the contribution owing to pure radiation damping is exact and determines the natural width of the line.

\section{Results}

The calculated lifetimes for energy levels in iron are given in table 1 , which is arranged in six columns as follows:

col. 1-electron configuration (with parent term)

col. 2 -term designation

col. $3-J$-value for the individual levels of the term col. 4-level value rounded to the nearest $\mathrm{cm}^{-1}$

col. 5-lifetime in nanoseconds given in most cases to three significant figures, although not more than two figures are meaningful. col. 6-the number of downward transitions contributing to the lifetime determination. 
TABLE 1. Radiative lifetimes for energy levels in neutral iron

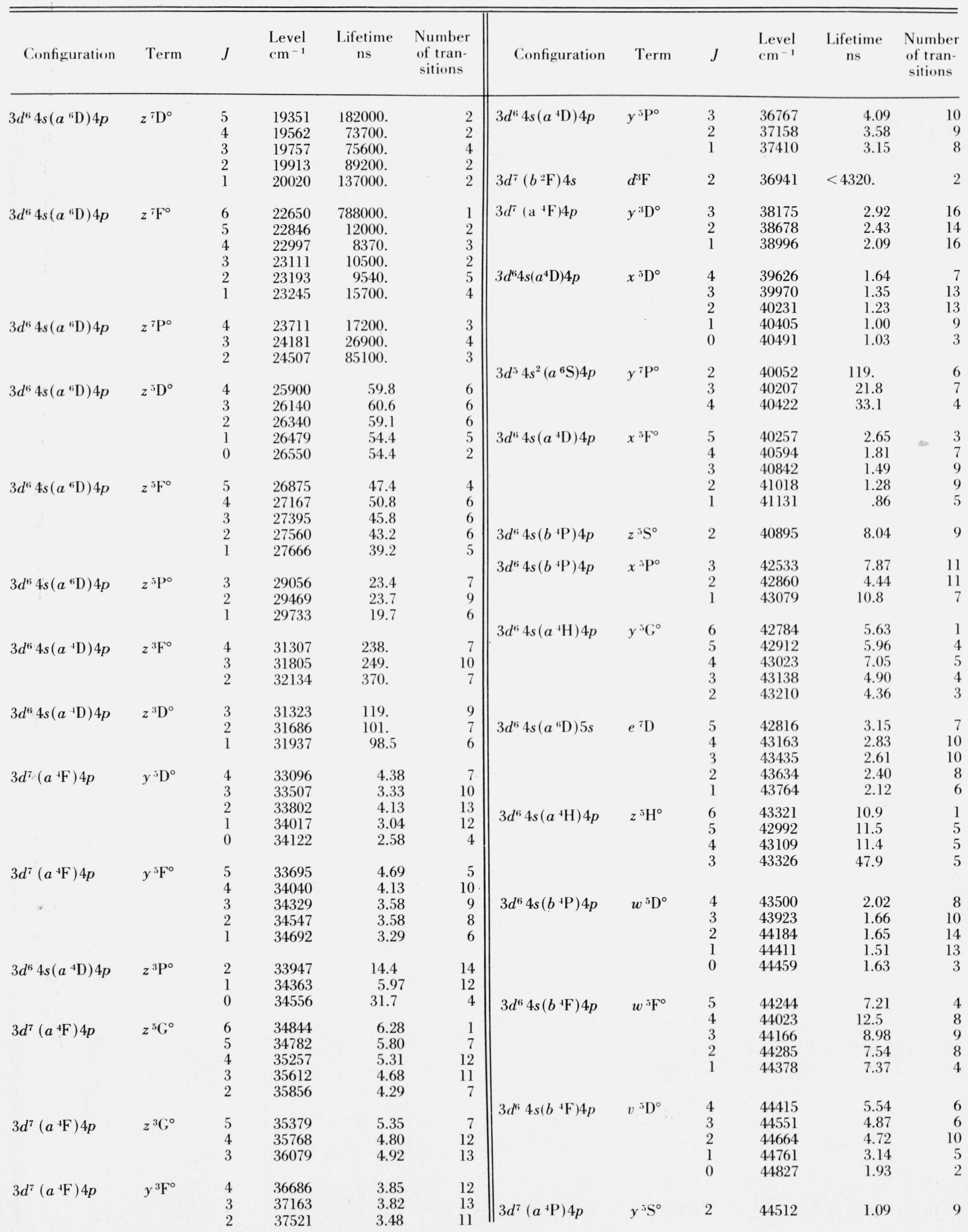


TABLE 1. Radiative lifetimes for energy levels in neutral iron-Continued

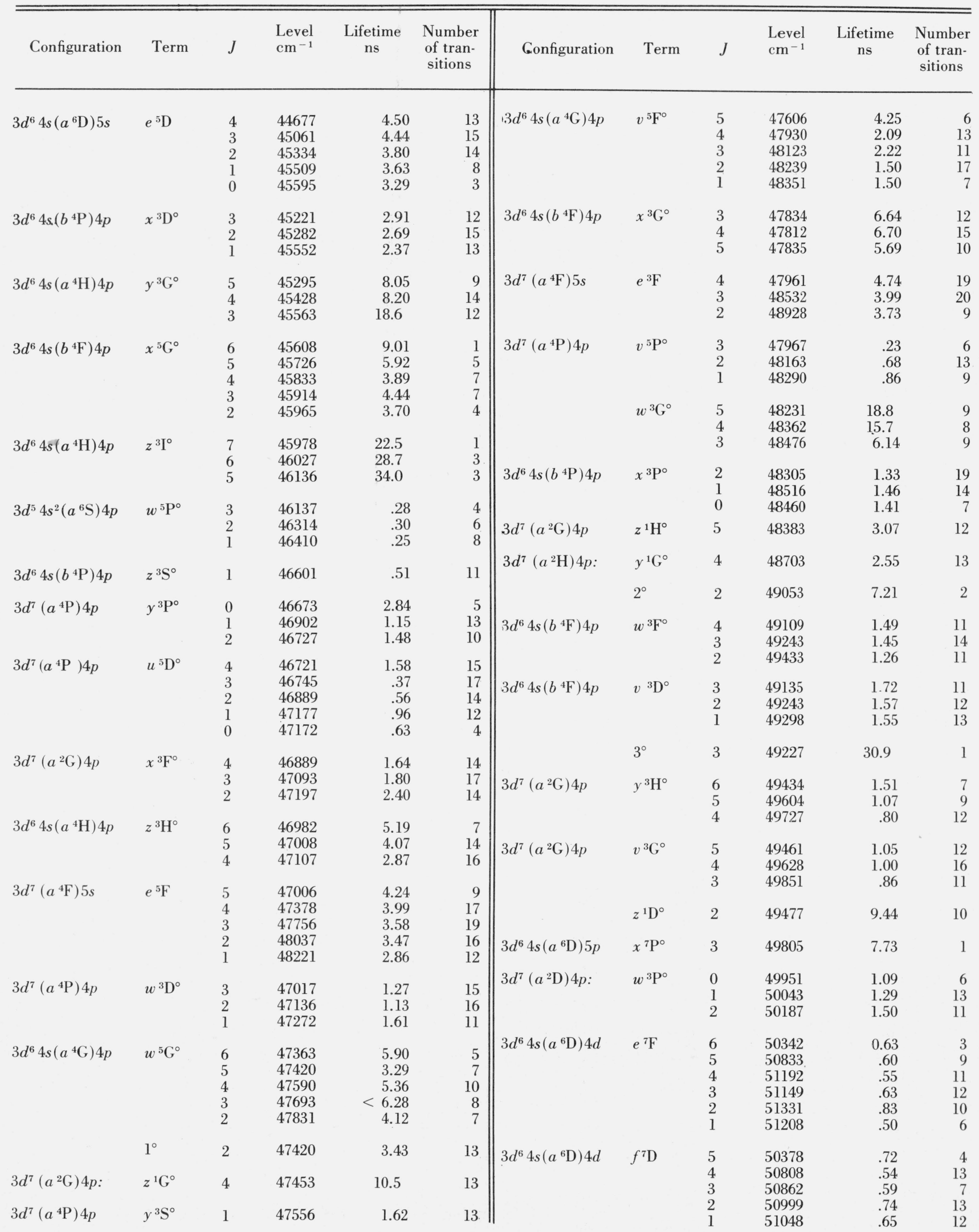


TABLE 1. Radiative lifetimes for energy Tevels in neutral iron-Continued

\begin{tabular}{|c|c|c|c|c|c|c|c|c|c|c|c|}
\hline Configuration & Term & $J$ & $\begin{array}{l}\text { Level } \\
\mathrm{cm}^{-1}\end{array}$ & $\begin{array}{l}\text { Lifetime } \\
\text { ns }\end{array}$ & $\begin{array}{l}\text { Number } \\
\text { of tran- } \\
\text { sitions }\end{array}$ & Configuration & Term & $J$ & $\begin{array}{l}\text { Level } \\
\mathrm{cm}^{-1}\end{array}$ & $\begin{array}{l}\text { Lifetime } \\
\text { ns }\end{array}$ & $\begin{array}{l}\text { Number } \\
\text { of tran- } \\
\text { sitions }\end{array}$ \\
\hline $3 d^{6} 4 s\left(a^{6} \mathrm{D}\right) 4 d$ & $f^{5} \mathrm{D}$ & $\begin{array}{l}4 \\
3 \\
2 \\
1 \\
0\end{array}$ & $\begin{array}{l}50423 \\
50534 \\
50699 \\
50880 \\
50981\end{array}$ & $\begin{array}{r}.80 \\
.75 \\
1.17 \\
1.25 \\
1.00\end{array}$ & $\begin{array}{r}14 . \\
17 \\
15 \\
10 \\
6\end{array}$ & $3 d^{6} 4 s\left(a^{6} \mathrm{D}\right) 4 d$ & $\begin{array}{l}5^{\circ} \\
e^{7} \mathrm{~S} \\
6^{\circ}\end{array}$ & $\begin{array}{l}3 \\
3 \\
5\end{array}$ & $\begin{array}{l}51436 \\
51570 \\
51630\end{array}$ & $\begin{array}{r}10.3 \\
.50 \\
3.47\end{array}$ & $\begin{array}{r}3 \\
11 \\
8\end{array}$ \\
\hline $3 d^{6} 4 s\left(a^{6} \mathrm{D}\right) 4 d$ & $e^{7} \mathrm{P}$ & $\begin{array}{l}4 \\
3 \\
2\end{array}$ & $\begin{array}{l}50475 \\
50611 \\
50861\end{array}$ & $\begin{array}{r}.86 \\
1.17 \\
1.93\end{array}$ & \begin{tabular}{r|}
8 \\
13 \\
9
\end{tabular} & $\begin{array}{l}3 d^{6} 4 s\left(a^{6} \mathrm{D}\right) 5 p \\
3 d^{7}\left(a^{2} \mathrm{P}\right) 4 p\end{array}$ & $\begin{array}{l}u^{5} \mathrm{P}^{\circ} \\
y^{1} \mathrm{D}^{\circ}\end{array}$ & $\begin{array}{l}3 \\
2\end{array}$ & $\begin{array}{l}51692 \\
51708\end{array}$ & $\begin{array}{l}71.1 \\
1.34\end{array}$ & $\begin{array}{r}1 \\
11\end{array}$ \\
\hline $3 d^{6} 4 s\left(a^{6} \mathrm{D}\right) 4 d$ & $e^{5} \mathrm{G}$ & $\begin{array}{l}6 \\
5 \\
4 \\
3 \\
2\end{array}$ & $\begin{array}{l}50523 \\
50704 \\
50980 \\
51219 \\
51370\end{array}$ & $\begin{array}{r}1.96 \\
1.30 \\
1.81 \\
.92 \\
1.14\end{array}$ & \begin{tabular}{r|}
6 \\
10 \\
10 \\
11 \\
12
\end{tabular} & $3 d^{6} 4 s\left(a^{6} \mathrm{D}\right) 4 d$ & $\begin{array}{l}7^{\circ} \\
x^{1} \mathrm{D}^{\circ} \\
e^{5} \mathrm{P}\end{array}$ & $\begin{array}{l}2 \\
2\end{array}$ & $\begin{array}{l}51756 \\
51762\end{array}$ & $\begin{array}{l}6.96 \\
5.07\end{array}$ & $\begin{array}{l}3 \\
6\end{array}$ \\
\hline $3 d^{7}\left(a^{2} \mathrm{G}\right) 4 p:$ & $z^{1} \mathrm{~F}^{\circ}$ & 3 & 50587 & 2.54 & 11 & $3 d^{6} 4 s\left(a^{6} \mathrm{D}\right) 4 d$ & $e^{5} \mathrm{P}$ & $\begin{array}{l}3 \\
2 \\
1\end{array}$ & $\begin{array}{l}51837 \\
52067 \\
52020\end{array}$ & $\begin{array}{l}2.44 \\
2.65 \\
2.71\end{array}$ & $\begin{array}{r}13 \\
15 \\
8\end{array}$ \\
\hline & $x^{1} \mathrm{G}^{\circ}$ & 4 & 50614 & 3.90 & 7 & $3 d^{7}\left(a^{2} \mathrm{P}\right) 4 p$ & $u^{3} \mathrm{D}^{\circ}$ & $\begin{array}{l}3 \\
2\end{array}$ & $\begin{array}{l}51969 \\
52297\end{array}$ & $\begin{array}{l}.81 \\
.47\end{array}$ & $\begin{array}{l}12 \\
11\end{array}$ \\
\hline $3 d^{6} 4 s\left(a^{6} \mathrm{D}\right) 4 d$ & $e^{7} \mathrm{G}$ & $\begin{array}{l}7 \\
6 \\
5 \\
4 \\
3 \\
2\end{array}$ & $\begin{array}{l}50652 \\
50968 \\
51229 \\
51335 \\
51461 \\
51540\end{array}$ & $\begin{array}{l}.74 \\
.91 \\
.89 \\
.79 \\
.57 \\
.58\end{array}$ & $\begin{array}{r}1 \\
4 \\
9 \\
13 \\
8 \\
6\end{array}$ & $3 d^{7}\left(a^{2} \mathrm{D}\right) 4 p$ & $t^{3} \mathrm{D}^{\circ}$ & $\begin{array}{l}1 \\
1 \\
1 \\
2 \\
3\end{array}$ & $\begin{array}{l}52512 \\
52181 \\
52683 \\
52213\end{array}$ & $\begin{array}{l}.38 \\
\\
1.97 \\
1.17 \\
1.09\end{array}$ & $\begin{array}{r}14 \\
8 \\
8 \\
11 \\
10\end{array}$ \\
\hline & & 1 & 51567 & .61 & 4 & $3 d^{7}\left(a^{2} \mathrm{H}\right) 4 p$ & $w^{3} \mathrm{H}^{\circ}$ & $\begin{array}{l}6 \\
5\end{array}$ & $\begin{array}{l}52431 \\
52613\end{array}$ & $\begin{array}{l}.96 \\
.84\end{array}$ & $\begin{array}{l}5 \\
6\end{array}$ \\
\hline $3 d^{6} 4 s\left(a^{6} \mathrm{D}\right) 5 p$ & $u^{5} \mathrm{~F}^{\circ}$ & $\begin{array}{l}5 \\
4 \\
3\end{array}$ & $\begin{array}{l}51017 \\
51381 \\
51619\end{array}$ & $\begin{array}{c}7.44 \\
6.09 \\
12.3\end{array}$ & $\begin{array}{l}2 \\
3 \\
3\end{array}$ & & & 4 & 52769 & 1.06 & 7 \\
\hline & & $\begin{array}{l}3 \\
2\end{array}$ & $\begin{array}{l}51619 \\
51828\end{array}$ & $\begin{array}{c}12.3 \\
2.14\end{array}$ & $\begin{array}{l}3 \\
4\end{array}$ & $3 d^{7}\left(a^{2} \mathrm{H}\right) 4 p$ & $y^{3} \mathrm{I}^{\circ}$ & $\begin{array}{l}7 \\
6 \\
5\end{array}$ & $\begin{array}{l}52655 \\
52514 \\
52899\end{array}$ & $\begin{array}{l}1.46 \\
1.24 \\
1.22\end{array}$ & $\begin{array}{l}2 \\
7 \\
6\end{array}$ \\
\hline $3 d^{6} 4 s\left(a^{4} \mathrm{G}\right) 4 p:$ & $x^{3} \mathrm{H}^{\circ}$ & $\begin{array}{l}6 \\
5\end{array}$ & $\begin{array}{l}51023 \\
51069\end{array}$ & $\begin{array}{l}6.97 \\
6.31\end{array}$ & $\begin{array}{r}7 \\
12\end{array}$ & $3 d^{7}\left(a^{2} \mathrm{P}\right) 4 p$ & $x^{3} \mathrm{~S}^{\circ}$ & 1 & 52858 & .46 & 11 \\
\hline $3 d^{6} 4 s\left(a^{6} \mathrm{D}\right) 5 p$ & $t^{5} \mathrm{D}^{\circ}$ & $\begin{array}{l}4 \\
3 \\
2 \\
1 \\
0\end{array}$ & $\begin{array}{l}51077 \\
51361 \\
51630 \\
51837 \\
51942\end{array}$ & $\begin{array}{r}<6.28 \\
2.73 \\
2.75 \\
8.01 \\
5.08\end{array}$ & $\begin{array}{l}2 \\
8 \\
6 \\
1 \\
1\end{array}$ & $3 d^{7}\left(a^{2} \mathrm{P}\right) 4 p$ & $\begin{array}{l}v^{3} \mathrm{P}^{\circ} \\
s^{3} \mathrm{D}^{\circ}\end{array}$ & $\begin{array}{l}2 \\
1 \\
3 \\
2\end{array}$ & $\begin{array}{l}52916 \\
53808 \\
52954 \\
53275\end{array}$ & $\begin{array}{r}.61 \\
.47 \\
\\
\\
3.94 \\
7.87\end{array}$ & $\begin{array}{r}9 \\
12 \\
\\
4 \\
2\end{array}$ \\
\hline $3 d^{6} 4 s\left(a^{6} \mathrm{D}\right) 4 d$ & $f^{5} \mathbf{F}$ & $\begin{array}{l}5 \\
4 \\
3 \\
2 \\
1\end{array}$ & $\begin{array}{l}51103 \\
51462 \\
51604 \\
51705 \\
51755\end{array}$ & $\begin{array}{l}1.28 \\
1.12 \\
2.09 \\
2.12 \\
3.45\end{array}$ & $\begin{array}{r}9 \\
11 \\
13 \\
12 \\
8\end{array}$ & $3 d^{7}\left(a^{4} \mathrm{~F}\right) 4 d$ & $g^{5} \mathrm{~F}$ & $\begin{array}{l}5 \\
4 \\
3 \\
2 \\
1\end{array}$ & $\begin{array}{l}53061 \\
53394 \\
53831 \\
54258 \\
54386\end{array}$ & $\begin{array}{l}2.95 \\
3.72 \\
4.21 \\
7.36 \\
1.84\end{array}$ & $\begin{array}{r}6 \\
11 \\
11 \\
6 \\
10\end{array}$ \\
\hline $3 d^{6} 4 s\left(a^{6} \mathrm{D}\right) 4 d$ & $e^{5} \mathrm{~S}$ & 2 & 51149 & 1.05 & 8 & $3 d^{7}\left(a^{2} \mathrm{H}\right) 4 p$ & $z^{1} \mathrm{I}^{\circ}$ & 6 & 53094 & 1.06 & 4 \\
\hline $3 d^{6} 4 s\left(a^{4} \mathrm{G}\right) 4 p$ & $v^{3} \mathrm{~F}^{\circ}$ & $\begin{array}{l}2 \\
3 \\
4\end{array}$ & $\begin{array}{l}51201 \\
51365 \\
51305\end{array}$ & $\begin{array}{l}2.47 \\
3.48 \\
2.05\end{array}$ & $\begin{array}{r}11 \\
9 \\
13\end{array}$ & $3 d^{7}\left(a^{4} \mathrm{~F}\right) 4 d$ & $h^{5} \mathrm{D}$ & $\begin{array}{l}4 \\
3 \\
2 \\
1\end{array}$ & $\begin{array}{l}53155 \\
53546 \\
53967 \\
54132\end{array}$ & $\begin{array}{r}4.39 \\
2.66 \\
2.73 \\
<5.26\end{array}$ & $\begin{array}{r}6 \\
11 \\
8 \\
6\end{array}$ \\
\hline $3 d^{6} 4 s\left(a^{4} \mathrm{D}\right) 5 s$ & $e^{3} \mathrm{D}$ & $\begin{array}{l}3 \\
2 \\
1\end{array}$ & $\begin{array}{l}51294 \\
51740 \\
52040\end{array}$ & $\begin{array}{l}2.56 \\
2.05 \\
2.64\end{array}$ & $\begin{array}{l}19 \\
19 \\
10\end{array}$ & $3 d^{7}\left(a^{4} \mathbf{F}\right) 4 d$ & $f^{5} \mathrm{P}$ & $\begin{array}{l}3 \\
2 \\
1\end{array}$ & $\begin{array}{l}53161 \\
53569 \\
53925\end{array}$ & $\begin{array}{r}<4.78 \\
2.09 \\
2.62\end{array}$ & $\begin{array}{r}4 \\
12 \\
11\end{array}$ \\
\hline $3 d^{6} 4 s\left(a^{4} \mathrm{D}\right) 5 s$ & $g{ }^{5} \mathrm{D}$ & $\begin{array}{l}4 \\
3 \\
2 \\
1 \\
0\end{array}$ & $\begin{array}{l}51351 \\
51771 \\
52050 \\
52214 \\
52257\end{array}$ & $\begin{array}{l}1.50 \\
1.57 \\
1.91 \\
1.52 \\
1.48\end{array}$ & $\begin{array}{r}19 \\
23 \\
23 \\
19 \\
6\end{array}$ & $3 d^{7}\left(a^{4} \mathbf{F}\right) 4 d$ & $f^{5} \mathrm{G}$ & $\begin{array}{l}6 \\
5 \\
4 \\
3 \\
2\end{array}$ & $\begin{array}{l}53169 \\
53282 \\
53769 \\
54161 \\
54376\end{array}$ & $\begin{array}{l}4.15 \\
1.82 \\
2.97 \\
2.02 \\
3.48\end{array}$ & $\begin{array}{r}5 \\
6 \\
12 \\
12 \\
5\end{array}$ \\
\hline \multirow[t]{2}{*}{$3 d^{7}\left(a^{2} \mathrm{H}\right) 4 p$} & $u^{3} \mathrm{G}^{\circ}$ & $\begin{array}{l}5 \\
4 \\
3\end{array}$ & $\begin{array}{l}51374 \\
51668 \\
51826\end{array}$ & $\begin{array}{r}1.11 \\
.80 \\
1.10\end{array}$ & $\begin{array}{l}11 \\
18 \\
10\end{array}$ & $3 d^{7}\left(a^{2} \mathrm{P}\right) 4 p$ & $z^{1} \mathrm{P}^{\circ}$ & 1 & 53230 & .57 & 11 \\
\hline & $4^{\circ}$ & 4 & 51409 & 2.79 & 11 & $3 d^{7}\left(a^{4} \mathrm{~F}\right) 4 d$ & $e^{5} \mathrm{H}$ & $\begin{array}{l}7 \\
6\end{array}$ & $\begin{array}{l}53275 \\
53353\end{array}$ & $\begin{array}{l}4.35 \\
3.79\end{array}$ & $\begin{array}{l}1 \\
2\end{array}$ \\
\hline
\end{tabular}


TABLE 1. Radiative lifetimes for energy levels in neutral iron-Continued

\begin{tabular}{|c|c|c|c|c|c|c|c|c|c|c|c|}
\hline Configuration & Term & $J$ & $\begin{array}{l}\text { Level } \\
\mathrm{cm}^{-1}\end{array}$ & $\begin{array}{l}\text { Lifetime } \\
\text { ns }\end{array}$ & $\begin{array}{l}\text { Number } \\
\text { of tran- } \\
\text { sitions }\end{array}$ & Configuration & Term & $J$ & $\begin{array}{l}\text { Level } \\
\mathrm{cm}^{-1}\end{array}$ & $\begin{array}{l}\text { Lifetime } \\
\text { ns }\end{array}$ & $\begin{array}{l}\text { Number } \\
\text { of tran- } \\
\text { sitions }\end{array}$ \\
\hline \multirow{5}{*}{$3 d^{6} 4 s\left(b^{4} \mathrm{D}\right) 4 p$} & & $\begin{array}{l}5 \\
4 \\
3\end{array}$ & $\begin{array}{l}53874 \\
54237 \\
54491\end{array}$ & $\begin{array}{l}3.21 \\
3.60 \\
3.72\end{array}$ & $\begin{array}{l}3 \\
3 \\
1\end{array}$ & & $s^{3} \mathrm{G}^{\circ}$ & $\begin{array}{l}5 \\
4 \\
3\end{array}$ & $\begin{array}{l}55907 \\
55906 \\
56098\end{array}$ & $\begin{array}{r}.74 \\
.65 \\
1.33\end{array}$ & $\begin{array}{l}7 \\
9 \\
3\end{array}$ \\
\hline & $9^{\circ}$ & 4 & 53329 & 3.35 & 5 & $3 d^{6} 4 s\left(b^{2} \mathrm{H}\right) 4 p$ & \multirow[t]{2}{*}{$u^{3} \mathrm{H}^{\circ}$} & $\begin{array}{l}6 \\
5\end{array}$ & $\begin{array}{l}56334 \\
56383\end{array}$ & $\begin{array}{l}.89 \\
.45\end{array}$ & $\begin{array}{l}4 \\
7\end{array}$ \\
\hline & \multirow[t]{2}{*}{$t^{5} \mathrm{P}^{\circ}$} & $\begin{array}{l}3 \\
2\end{array}$ & $\begin{array}{l}53389 \\
54112\end{array}$ & $\begin{array}{l}9.82 \\
1.13\end{array}$ & $\begin{array}{l}1 \\
3\end{array}$ & \multirow[b]{2}{*}{$3 d^{6} 4 s\left(a^{6} \mathrm{D}\right) 5 d$} & & 4 & 56423 & .46 & 9 \\
\hline & & 1 & 54271 & 1.98 & 6 & & 1 & 5 & 56428 & .91 & 4 \\
\hline & $y^{1} \mathrm{~F}^{\circ}$ & 3 & 53661 & 1.94 & 9 & $3 d^{6} 4 s\left(a^{6} \mathrm{D}\right) 5 d$ & 2 & 4 & 56452 & 1.29 & 5 \\
\hline $3 d^{7}\left(a^{2} \mathrm{H}\right) 4 p$ & $y^{1} \mathrm{H}^{\circ}$ & 5 & 53722 & 1.69 & 2 & \multirow[t]{2}{*}{$3 d^{7}\left(a^{2} \mathrm{D}\right) 4 p$} & $u^{3} \mathrm{~F}^{\circ}$ & $\begin{array}{l}4 \\
3\end{array}$ & $\begin{array}{l}56593 \\
56783\end{array}$ & $\begin{array}{l}.52 \\
.53\end{array}$ & $\begin{array}{l}6 \\
6\end{array}$ \\
\hline $3 d^{7}\left(a^{4} \mathrm{~F}\right) 4 d$ & $e^{3} \mathrm{G}$ & $\begin{array}{l}5 \\
4\end{array}$ & $\begin{array}{l}53739 \\
54067\end{array}$ & $\begin{array}{l}2.28 \\
2.79\end{array}$ & $\begin{array}{r}12 \\
9\end{array}$ & & & 2 & 56859 & .44 & 7 \\
\hline & & 3 & 54379 & 3.71 & 3 & $3 d^{6} 4 s\left(a^{6} \mathrm{D}\right) 5 d$ & 3 & 4 & 56843 & 1.02 & 5 \\
\hline $3 d^{7}\left(a^{4} \mathbf{F}\right) 4 d$ & $f^{3} \mathrm{D}$ & 3 & 53748 & 2.66 & $\begin{array}{l}12 \\
14\end{array}$ & & $v^{1} \mathrm{G}^{\circ}$ & 4 & 56951 & .42 & 5 \\
\hline & & 1 & 54449 & $\begin{array}{r}2.21 \\
.99\end{array}$ & 14 & $3 d^{6} 4 s\left(b^{2} \mathrm{H}\right) 4 p$ & $x^{3} \mathrm{I}^{\circ}$ & 7 & 57028 & .65 & 2 \\
\hline & $x^{1} \mathrm{~F}^{\circ}$ & 3 & 53763 & 1.57 & 10 & & & $\begin{array}{l}6 \\
5\end{array}$ & $\begin{array}{l}57070 \\
57104\end{array}$ & $\begin{array}{l}.70 \\
.73\end{array}$ & $\begin{array}{l}3 \\
3\end{array}$ \\
\hline $3 d^{6} 4 s\left(a^{6} \mathrm{D}\right) 6 s$ & $g{ }^{7} \mathrm{D}$ & 5 & 53801 & 3.90 & 4 & & $t^{3} \mathrm{~F}^{\circ}$ & 4 & 57550 & .51 & 5 \\
\hline & & $\begin{array}{l}4 \\
3\end{array}$ & $\begin{array}{l}54125 \\
54414\end{array}$ & 2.23 & 6 & & & $\begin{array}{l}3 \\
2\end{array}$ & $\begin{array}{l}57641 \\
57709\end{array}$ & $\begin{array}{r}1.26 \\
.44\end{array}$ & $\begin{array}{l}8 \\
5\end{array}$ \\
\hline & & 2 & 54612 & $<3.98$ & 4 & & & & & & \\
\hline & & 1 & 54748 & 3.30 & 3 & $3 d^{6} 4 s\left(a^{4} \mathrm{D}\right) 4 d$ & $i^{5} \mathrm{D}$ & $\begin{array}{l}4 \\
3\end{array}$ & $\begin{array}{l}57698 \\
57814\end{array}$ & $\begin{array}{l}.59 \\
.56\end{array}$ & $\begin{array}{l}7 \\
8\end{array}$ \\
\hline $3 d^{7}\left(a^{4} \mathbf{F}\right) 4 d$ & $e^{3} \mathrm{H}$ & 6 & 53841 & 3.51 & 2 & & & 2 & 57974 & .49 & 9 \\
\hline & & $\begin{array}{l}5 \\
4\end{array}$ & $\begin{array}{l}54267 \\
54555\end{array}$ & $\begin{array}{c}19.6 \\
2.90\end{array}$ & $\begin{array}{l}1 \\
4\end{array}$ & $3 d^{6} 4 s\left(a^{6} \mathrm{D}\right) 7 s$ & $h^{7} \mathrm{D}$ & 5 & 57897 & 2.75 & 2 \\
\hline $3 d^{6} 4 s\left(b^{4} \mathrm{D}\right) 4 d:$ & $10^{\circ}\left({ }^{5} \mathrm{D}^{\circ} ?\right)$ & 3 & 53892 & 1.18 & 9 & $3 d^{6} 4 s\left(a^{4} \mathrm{D}\right) 4 d$ & $g^{5} \mathrm{G}$ & 6 & 58002 & .50 & 3 \\
\hline $3 d^{6} 4 s\left(a^{4} \mathrm{G}\right) 4 p$ & $t^{3} \mathrm{G}^{\circ}$ & 5 & 53983 & .67 & 8 & & & 4 & $\begin{array}{l}50212 \\
58520\end{array}$ & $\begin{array}{l}1.00 \\
2.17\end{array}$ & $\begin{array}{l}4 \\
5\end{array}$ \\
\hline & & 4 & 54237 & .78 & 11 & & & 3 & 58710 & $<8.82$ & 2 \\
\hline & & 3 & 54600 & .67 & 13 & & & & 58825 & 2.10 & 3 \\
\hline & $11^{\circ}$ & 3 & 54005 & 9.69 & 7 & $3 d^{6} 4 s\left(a{ }^{4} \mathrm{D}\right) 4 d$ & 4 & 2 & 58213 & .71 & 7 \\
\hline $3 d^{6} 4 s\left(b^{4} \mathrm{D}\right) 4 d$ & $12^{\circ}\left({ }^{5} \mathrm{~F}^{\circ} ?\right)$ & 5 & 54014 & 1.77 & 5 & & $r^{3} \mathrm{G}^{\circ}$ & 5 & 59927 & .37 & 1 \\
\hline & $13^{\circ}$ & 4 & 54301 & 2.05 & 9 & & & 3 & 60365 & .09 & 2 \\
\hline $3 d^{7}\left(a^{4} \mathrm{~F}\right) 4 d$ & $f^{3} \mathrm{~F}$ & 4 & 54683 & 3.62 & 9 & & $t^{3} \mathrm{H}^{\circ}$ & 6 & 60366 & .17 & 4 \\
\hline & & 3 & 55125 & 2.57 & 9 & & & 4 & $\begin{array}{r}60549 \\
60750\end{array}$ & .16 & $\begin{array}{l}4 \\
5\end{array}$ \\
\hline & & 2 & 55379 & 2.15 & 6 & & & 4 & 60758 & .18 & 5 \\
\hline & $w^{1} \mathrm{G}^{\circ}$ & 4 & 54811 & 2.94 & 4 & & $q^{3} \mathrm{G}^{\circ}$ & 3 & 60807 & .32 & 4 \\
\hline $3 d^{7}\left(a^{4} \mathrm{~F}\right) 4 d$ & $e^{3} \mathrm{P}$ & 2 & 54880 & 3.85 & 5 & & & 3 & 53358 & 3.88 & 7 \\
\hline & & 1 & 55376 & 2.47 & 5 & & & $\begin{array}{l}4 \\
3\end{array}$ & $\begin{array}{l}53610 \\
53734\end{array}$ & $\begin{array}{l}7.73 \\
8.45\end{array}$ & $\begin{array}{l}3 \\
6\end{array}$ \\
\hline & & 0 & 55727 & 1.08 & 2 & & & 2 & 53749 & 2.88 & 6 \\
\hline & $v^{3} \mathrm{H}^{\circ}$ & 4 & 55446 & .88 & 6 & & & 3 & $\begin{array}{l}53785 \\
53892\end{array}$ & 6.62 & 3 \\
\hline & & 5 & 55430 & .74 & 9 & & & $\begin{array}{l}4 \\
3\end{array}$ & $\begin{array}{l}53882 \\
54289\end{array}$ & $\begin{array}{l}.13 \\
4.58\end{array}$ & $\begin{array}{l}4 \\
7\end{array}$ \\
\hline & & 6 & 55490 & .66 & 5 & & & 3 & 54357 & 2.30 & 7 \\
\hline & $x^{1} \mathrm{H}^{\circ}$ & 5 & 55526 & 1.96 & 5 & & & $\begin{array}{l}3 \\
3\end{array}$ & $\begin{array}{l}57565 \\
60564\end{array}$ & $\begin{array}{r}2.48 \\
93\end{array}$ & $\begin{array}{l}4 \\
5\end{array}$ \\
\hline $3 d^{7}\left(a^{2} \mathrm{D}\right) 4 p:$ & $w^{1} \mathrm{D}^{\circ}$ & 2 & 55754 & .17 & 8 & & & 2 & 62081 & 1.14 & 3 \\
\hline & $w^{1} \mathrm{~F}^{\circ}$ & 3 & 55791 & .74 & 8 & Fe II $\left(a^{6} \mathrm{D}_{4_{\frac{1}{2}}}\right)$ & Limit & & 63700 & & \\
\hline
\end{tabular}


In pure $L S$ coupling the lowest three terms in table 1 would be metastable. This metastability is clearly exhibited in our results for the lifetimes of these levels. Though finite because of slight departures from $L S$ coupling, the lifetimes obtained for these levels are nevertheless several orders of magnitude longer than those obtained for the nonmetastable levels. Another point of interest is the marked tendency throughout the table towards equality in the lifetimes of the individual levels of a single term, as expected.

Although the 3300 lines entering into these determinations represent more than three quarters of the classified lines observed in the laboratory, and the strongest ones at that, there are a few cases where the values stated for the lifetimes must be considered as an upper limit. For example, the $d^{3} \mathrm{~F}_{2}$ level at 36941 $\mathrm{cm}^{-1}$, which is the lowest even level in our list, must make its strongest downward combinations in the infrared, beyond the range of our list of lines. For a few other levels, lines of significant intensity lacked reliable values of $\log g f$. Where known, such values are marked with the "less than" $(<)$ symbol.

Of the 408 lifetimes reported in table 1 , three have been measured elsewhere by more direct methods. Karstensen and Richter [1965] measured lifetimes for $z^{3} \mathrm{~F}_{4}^{\circ}$ and $z^{3} \mathrm{D}_{1}^{\circ}$ by a delayed coincidence method in a hollow cathode and Otten and Wagner [1967] measured the lifetime for $z^{5} \mathbf{F}_{5}^{\circ}$ by a double resonance experiment in an atomic beam. The lifetimes in nanoseconds are compared below.

$\begin{array}{ccc}\text { Level } & \text { This Paper } & \text { Elsewhere } \\ z^{5} \mathrm{~F}_{5}^{\circ} & 47 & 54 \\ z^{3} \mathrm{~F}_{4}^{\circ} & 238 & 220 \\ z^{3} \mathrm{D}_{1}^{\circ} & 98 & 100\end{array}$

A detailed discussion of the accuracy of the absolute scale of the oscillator strengths from which our lifetimes are derived is given in section 5 of Corliss and
Warner [1966]. From that discussion we conclude that our general scale of lifetimes may be small by about $30 \pm 10$ percent. In the comparison of values for the three levels shown in the preceding paragraph our scale is small by about 3 percent, which is well within the uncertainty of the individual values. This latter uncertainty cannot be precisely evaluated, but may be about 50 percent.

The work reported in this paper is based on the work originally conceived by the late W. F. Meggers and brought to fruition in his monumental "Tables of Spectral-Line Intensities," which was published in 1961 as NBS Monograph 32.

The present authors were privileged to be associated for many years with Dr. Meggers. His encouragement and good counsel were always a source of inspiration to us.

\section{References}

Corliss, C. H. and Bozman, W. R. (1962), Experimental transition probabilities for spectral lines of seventy elements, NBS Mono. 53 (July 20, 1962).

Corliss, C. H. and Tech, J. L. (1967), Oscillator strengths and transition probabilities for 3328 Lines of Fe I. NBS Mono. 108.

Corliss, C. H. and Warner, B. (1964), Ap. J. Suppl. 8, 395, No. 83.

Corliss, C. H. and Warner, B. (1966), J. Res. NBS 70A (Phys. and Chem.), No. 4, 325.

Einstein, A. (1917), Phys. Zeits. 18, 121.

Huber, M. and Tobey, F. L. (1967), Shock Tube Spectroscopy Laboratory, Scientific Report No. 16, Harvard College Observatory. Also Astrophys. J. (in press).

Karstensen, F. and Richter J. (1965), New Method of Measuring Transition Probabilities, AF EOAR Grant 63-110.

Mitchell, A. G. C. and Zemansky, M. W., Resonance Radiation and Excited Atoms, p. 145 (Cambridge University Press, 1934).

Otten, E. W. and Wagner, R. (1967), Physica 33, 283.

Warner, B. and Cowley, C. R. (1967), J. Quant. Spectrosc. Radiative Transfer 7, 751 .

(Paper 71A6-472) 